\title{
Human Computer Interaction Model based Virtual Whiteboard: A Review
}

\author{
Aniket E. Kudale \\ Department of Computer Engineering \\ Vishwakarma Institute of Information Technology, \\ Kondhwa, Pune - 411048, \\ Savitribai Phule Pune University, India
}

\author{
K.H. Wanjale \\ Department of Computer Engineering \\ Vishwakarma Institute of Information Technology, \\ Kondhwa, Pune - 411048, \\ Savitribai Phule Pune University, India
}

\begin{abstract}
Interactive whiteboard involving human interaction have been an active field of research in past years in human computer interaction (HCI). There is a need for adoption of natural interfaces between user and computing devices. This is trending in educational as well as corporate sector where various interactive application, tools and methodologies are used to make the presentation, summarization of difficult concepts easy and also to simulate collaborative work and enhance teaching practices. For Instance, it is a common practice to draw business processes on a traditional whiteboard, using conventional modeling notation such as UML, BPMN, during meetings between software programmers and business clients. Since it is a traditional whiteboard, software developer may need to redesign the process diagram on his computer by referring the whiteboard, this can be time consuming. Alternative way will be to use pen-based input device such as an electronic interactive whiteboard or graphics tablet. Use and working of interactive whiteboard have received researchers' attention as it has been found to be easy and highly effective interaction method. This paper presents a short review for interactive whiteboard technology and its operations proposed in the literature. Some examples of applications are also investigated.
\end{abstract}

\section{General Terms}

Human Factors, Human Computer Interaction

\section{Keywords}

Interactive whiteboard, Human Computer Interaction, User Interface, Virtual Whiteboard, Interactive Learning Environment

\section{INTRODUCTION}

Interactive whiteboard (IWB) are becoming more popular in educational as well as corporate settings [8]. Interactive whiteboard is a system which displays a computer screen on large screen or surface which is made touch enabled [1]. User can touch and interact with the screen by using either their hand or stylus pen. This enables user to perform normal computer operation (moving the cursor, clicking, drawing, writing etc.) on screen just by touching the surface. Basically, it replaces the traditional blackboard or whiteboard with interactive surface. Hence user can annotate the projected screen as if it is written on a blackboard [3]

All Interactive whiteboards are touch sensitive boards which generally operate through projector-camera setup connected to a computing system [4][5]. A typical interactive whiteboard setup consists of four main components; a computer, digital projector, device for interaction and software tool which is compatible with the operating system and hardware. In this, interaction can be provided in different ways such as optical, capacitive, resistive, electromagnetic, and ultrasonic. These methods or techniques are divided into two categories as active marker and passive marker. Marker is used for interaction with the board surface. Active markers are the source of signal such as light, have good stability e.g. Infrared LED, Laser pointer. Passive markers are wide range of reflective markers, which are unstable for signal detection. E.g. Retro-reflective tape, color tape, color token etc. Active markers are expensive but have high detection rate and passive markers has low cost and low detection rate.

Mostly the interactive whiteboard are controlled by using hand/finger [12][7], stylus, pen [2] etc. Also natural interaction can be easily associated with the system [7]. Natural interaction means capturing the [18] body movements/gesture of the user in order to control computer.

This paper presents a short survey of the work done in interactive whiteboard technologies. The remainder of the paper is organized as follows. Section II describes the hardware setup for typical interactive whiteboard. Section III summarizes the different operating modes of interactive whiteboards. Section IV summarizes the related works of touch based interactive whiteboard systems. Section V, discusses the application of interactive whiteboard in educational and corporate settings followed by conclusion in Section VI

\section{HARDWARE SETUP OF INTERACTIVE WHITEBOARD}

Hardware setup of an interactive projection system consists of a computer, projector, a sensing device (such as webcam, Wii Remote, Kinect or other similar devices) and a marker (such as pen, stylus) to interact with the system. Some modern interactive whiteboard also allow user to use body movement/gesture for interaction.

Here the projector projects [4] the computer screen on flat surface such as wall, whiteboard. The sensing device such as webcam captures the movement made by pen, stylus or hand [12], and then the computer analyzes the captured movement and interprets operations on computer [6].

The basic framework of interactive projection system is presented in Figure. 1. Here we can see computer running software, a webcam which acts as a sensing device which allows us to capture the interaction made on the projected surface and an infrared pointer pen is used to generate interaction and a LCD projector to project the computer screen over the whiteboard. 


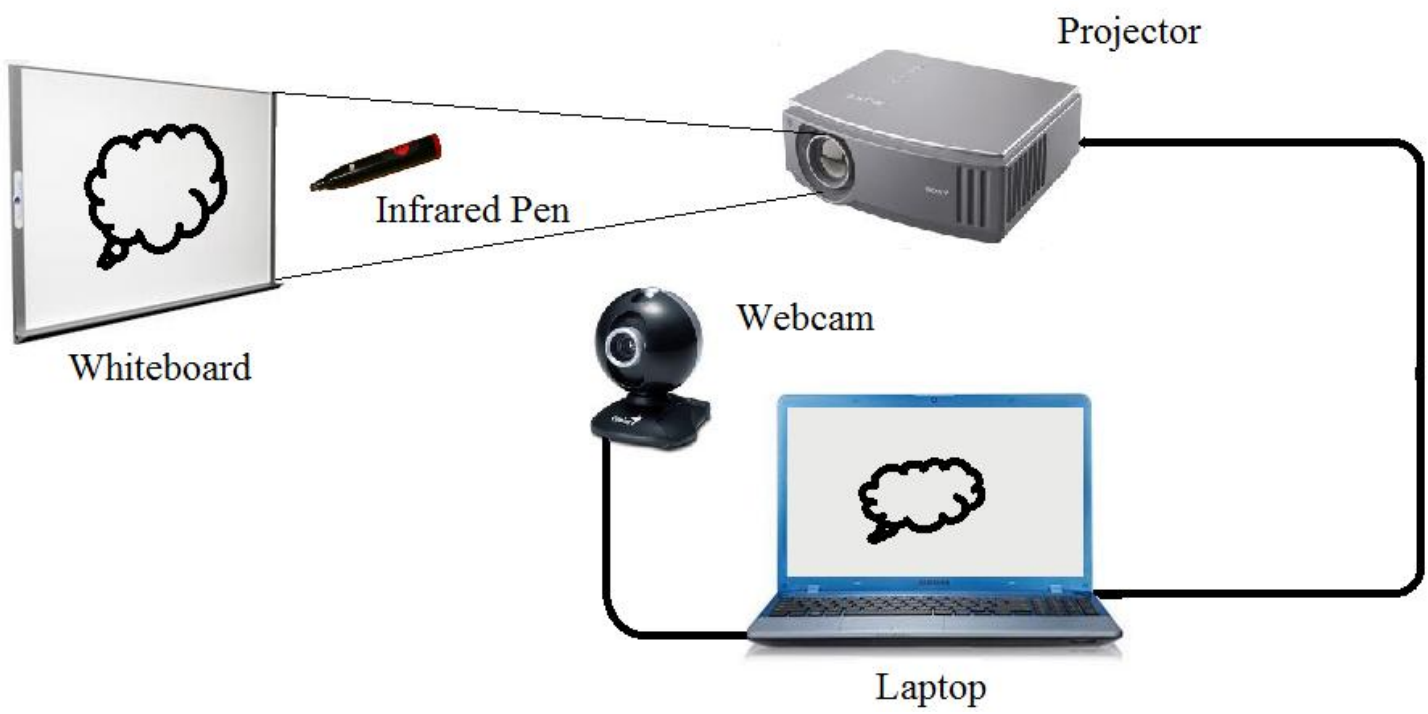

Fig 1: Basic Framework of Interactive Projection System

Generally, the projector and camera are fixed. The computer screen can be projected on wall, whiteboard or any other flat surface by adjusting the projection angle of the projector. Since the size of the screen is adjustable, it satisfies the most of the applications' requirement.

\section{DIFFERENT TYPES OF INTERACTIVE WHITEBOARD}

Interactive whiteboard is generally connected to computer through USB or serial cable, or by Bluetooth [17]. The computer content is projected on wall surface or whiteboard using a projector. After this a pointer or device may be used to interact or control the projected content. This reduces the need to go to computer keyboard and mouse to enter text or move cursor.

\subsection{Infrared scan based whiteboard}

An infrared based whiteboard is an interactive display that is connected to computer and projector. The board is mounted on wall or on stand. The infrared sensor is attached to the board which senses the movement of pen, finger or other devices over the projected image on board. The movement is captured by infrared light interference created at the surface when user touches the surface. When the surface is touched, triangulation principle locates the position of the marker, stylus or finger [9].

\subsection{Resistive touch based interactive whiteboard}

This type of interactive whiteboard requires a pointing device such as stylus, pen or user can use their finger to interact with the board. The resistive touch based interactive whiteboard consists of a membrane which is stretched over the surface which when pressed makes contact with the conductive material. The touch point position is located electronically and can be configured to perform some operations. These whiteboard are easy to use, but are costly [3].

\subsection{Electromagnetic pen based interactive whiteboard}

This kind of interactive whiteboard consists of an array of wires which are typically embedded behind the surface of board that comes in contact with the stylus, which has a coil in its tip which determines the $\mathrm{X}$ and $\mathrm{Y}$ coordinates of stylus on board. The stylus is passive it does not contain battery or power source; it just alters the electrical signal when touched to board.

\subsection{Wii remote based interactive whiteboard}

In this, interactive whiteboard is emulated using Wii remote and an infrared pen [16][17][13]. The Wii-based interactive whiteboard was invented by Johnny Lee, in 2007 . The setup consists of a computer, projector, a Wii remote and an infrared pen. The Wii remote is connected to computer via Bluetooth, Wii remote has IR camera which acts as a tracking device which senses Infrared light emitting from an infrared pen. The projector projects the computer content on board surface and then user uses the infrared pen as a pointer to operate or control the content on the board surface. This approach is user friendly, highly flexible and moderately inexpensive.

\subsection{Interactive whiteboard via an interactive projector}

In this kind of system a CMOS camera is built within a projector, which acts as sensor. The projector projects the computer content, and the camera detects the position of an active IR pen when it comes in contact with the surface of the board on which content is projected. This system generally costs more compared to other system. It has some operating problems such as problem caused by 'loss of line of sight' between the stylus/pen and the projector/camera sensor.

\section{RELATED WORK}

In recent years, there has been lots of research going on in developing interactive whiteboard surfaces.

$\mathrm{Hu}$, Jun, et al. [1] proposed a novel approach which enables finger touch interaction by detecting touch event on projected surface based on button distortion. This requires a standard camera and a projector, and can be used to emulate interactive whiteboard based on finger touch.

Chen Rui, et al, [2] proposed an IR pen-based table top system that uses Wii-remote to track the IR pen movement and thus interact with the projected content. In this system they developed a novel method allowing multiple IR pens to be detected and tracked simultaneously at the same time. 
Dai, Jingwen, et al. [5] proposed a system that lets any table top surface to which content is projected to be a touch enabled surface, using just a projector and a camera. Touch is detected by using computer vision algorithms on hand/finger such as hand segmentation and tracking.

Soares Christope, et al. [6] proposed a simple camera, projector system and an IR pen for interactive whiteboard operation. They used webcam with photographic film attached to it, in order to detect IR pen light. This system however faced accuracy issue as background noise disturbs the functioning and also affects its efficiency.

Chien-Yu Lin, et al. [8] discussed the application of Wiiremote based interactive surface in educational settings

Michal Lech, et al. [9] developed a system which allows interactive whiteboard to be controlled using hand gesture of user. Presented a hardware and software on virtual whiteboard with special focus on utilizing the fuzzy rule-based gesture recognition concept and kalman filters for the prediction of consecutive hand positions also evaluated their system for efficacy.

Shuai Zhang, et al. and Marco Ronchetti, [10][11] proposed interactive whiteboard setup using Kinect gaming system as a sensing device. Kinect is connected to computer which acts as a sensing device to locate the pointer such as hand, IR pen on board on which the content is projected. Problem with such system is the cost of the Kinect device.

Massimo Bosetti, et al, [13] presented a result analysis on usage Wii-remote based interactive whiteboard by conducting a survey in educational institutes to understand various concerns and issues regarding usage of Wii-remote for interactive whiteboard.

Pranav Mistry, [14] introduced wearable gesture interface. It lets user to project content on any surface and user can interact with it using hand gesture [15]. A marker such as a color tape is wrapped around the fingers and a portable projector camera setup sense the color marker gesture made by fingers [19] to interact with the board.

Johnny Chung Lee, [16] [17] proposed the first Wii-remote based interactive whiteboard. The system consist of a projector, a computer, a Wii-remote connected to computer via Bluetooth; this acts as a sensing device, an Infrared pen is used as a pointer; to interact with the projected content on board surface.

Andrew Wilson, [20] proposed a compact interactive tabletop projection vision system. It uses camera-projector pair. Introduced touch detection algorithm based on the observation of shadows also used an optical flow algorithm for interacting with onscreen information.

Franois Brard, [21] emulated interactive whiteboard using camera projector pair. Colored plastic tokens were used to interact with the projected content on board surface. Color detection algorithms are used in the system to detect the position of color tokens on surface of whiteboard.

\section{APPLICATIONS OF INTERACTIVE WHITEBOARDS}

The interactive whiteboards are used in variety of settings, including corporate meeting rooms, in classrooms at all levels of education, at work groups, in training room, broadcasting studios, and others.

\subsection{In Classrooms}

Interactive whiteboards have replaced traditional blackboards, whiteboards, or other video/media systems combination. With interactive whiteboard a presenter or teacher can teach more interactively and can work with multimedia features, which helps in better understanding of the content been presented. User can interact; write or draw anything on the board surface which is digitally captured, saved and can be shared with students for reviewing. The use of interactive whiteboard can increase the student interest in learning activities in classroom [8].

\subsection{In Corporate Meeting Rooms}

Interactive whiteboards can be used to explain information and statistics interactive at a business meeting [21]. It displays all relevant information in an interesting format. The presenter can manipulate the content being shown interactively. Interactive whiteboard is a effective tool or system in the business world. The information such as graphs, charts, bars, sales number or any other statistical information can be delivered during meetings for effective communication between presenter and attendees

\section{CONCLUSION}

This paper exposes various interactive whiteboards, which replaces the traditional blackboards and whiteboards. Related work has been discussed for interactive whiteboard which use commodity hardware, along with the application of it in educational and corporate setting. Interactive whiteboard have become the basic and essential part for digital instruction.

\section{REFERENCES}

[1] $\mathrm{Hu}$, Jun, et al. "Bare-fingers Touch Detection by the Button's Distortion in Projector-camera System.", IEEE Transactions on Circuits and Systems for Video Techmology, vol. 24, no. 4, pp. 566-575, April 2014.

[2] Chen, Rui, Po-Jui Ray Chen, Rui Feng, Yilin Elaine Liu, Andy $\mathrm{Wu}$, and Ali Mazalek. "SciSketch: a tabletop collaborative sketching system." In Proceedings of the 8th International Conference on Tangible, Embedded and Embodied Interaction, pp. 247-250. ACM, Feb. 2014.

[3] Şimşek, Serdar, and Pınar Onay Durdu. "Developing an Interactive Learning Environment with Kinect." In HCI International 2014-Posters' Extended Abstracts, pp. 150155. Springer International Publishing, 2014.

[4] Amano, Toshiyuki. "Projection Center Calibration for a Co-located Projector Camera System." Computer Vision and Pattern Recognition Workshops (CVPRW) IEEE Conference, pp. 449-454, June. 2014

[5] Dai, Jingwen, and C-KR Chung. "Touchscreen Everywhere: On Transferring a Normal Planar Surface to a Touch-Sensitive Display.", IEEE Transaction on Cybernetics, vol. 44, no. 8, pp. 1383-1396, Aug. 2014.

[6] Soares, Christophe, Rui S. Moreira, José M. Torres, and Pedro Sobral. "LoCoBoard: Low-Cost Interactive Whiteboard Using Computer Vision Algorithms." ISRN Machine Vision, vol. 2013.

[7] Al-Rahayfeh, A. M. E. R., and M. I. A. D. Faezipour. "Eye Tracking and Head Movement Detection: A Stateof-Art Survey."IEEE Journal of Translational Engineering in Health and Medicine, vol. 1, Nov. 2013.

[8] Lin, Chien-Yu, Ming Chi Lin, Li-Jun Shih, and WeiHsuan Hu. "Application of low-cost interactive floors on 
special education and assistive technology." International Journal of Elementary Education, vol. 2, no. 1, pp. 1-7, 2013.

[9] Lech, Michal, Bozena Kostek, and Andrzej Czyzewski. "Virtual Whiteboard: A gesture-controlled pen-free tool emulating school whiteboard." Intelligent Decision Technologies, vol. 6, no. 2, pp. 161-169, Feb. 2012.

[10] Ronchetti, Marco, and Mattia Avancini. "Using Kinect to emulate an Interactive Whiteboard." MS in Computer Science, University of Trento, 2011.

[11] Zhang, Shuai, Wenrui He, Qiao Yu, and Xiaojuan Zheng. "Low-cost interactive whiteboard using the Kinect." International Conference on Image Analysis and Signal Processing (IASP), pp. 1-5. 2012.

[12] Rautaray, Siddharth S., and Anupam Agrawal. "Real time hand gesture recognition system for dynamic applications." International Journal of UbiComp, vol. 3, no. 1, pp. 21-31, Jan 2012.

[13] Bosetti, Massimo, Pietro Pilolli, Matteo Ruffoni, and Marco Ronchetti. "Interactive whiteboards based on the WiiMote: Validation on the field." 14th International Conference on Interactive Collaborative Learning (ICL), pp. 269-273, Sept. 2011

[14] Lech, Michal, and Bozena Kostek. "Gesture-based computer control system applied to the interactive whiteboard." 2nd International Conference on Information Technology (ICIT), pp. 75-78, Jun. 2010.

[15] Mistry, Pranav, Pattie Maes, and Liyan Chang. "WUWwear Ur world: a wearable gestural interface." In CHI'09 extended abstracts on Human factors in computing systems, pp. 4111-4116. ACM, Apr. 2009.

[16] Schreiber, Michael, Margeritta von WilamowitzMoellendorff, and Ralph Bruder. "New interaction concepts by using the wii remote." In Human-Computer Interaction. Novel Interaction Methods and Techniques, pp. 261-270. Springer Berlin Heidelberg, 2009.

[17] Lee, Johnny Chung. "Hacking the nintendo wii remote." Pervasive Computing, IEEE, vol.7, no. 3, pp. 39-45, Jul 2008.

[18] Pallejà, Tomàs, Edgar Rubión Soler, Mercè Teixido, Marcel Tresanchez, Alicia Fernández del Viso, Carlos Rebate Sánchez, and Jordi Palacin. "Using the Optical Flow to Implement a Relative Virtual Mouse Controlled by Head Movements." Journal of Universal Computer Science, vol. 14, no. 19, pp. 3127-3141, Nov. 2008

[19] Argyros, Antonis A., and Manolis IA Lourakis. "Visionbased interpretation of hand gestures for remote control of a computer mouse." In Computer Vision in HumanComputer Interaction, pp. 40-51. Springer Berlin Heidelberg, 2006.

[20] Wilson, Andrew D. "PlayAnywhere: a compact interactive tabletop projection-vision system." In Proceedings of the 18th annual ACM symposium on User interface software and technology, pp. 83-92. ACM Oct. 2005.

[21] Bérard, François. "The magic table: Computer-vision based augmentation of a whiteboard for creative meetings." In IEEE workshop on Projector-Camera Systems. Oct. 2003. 